\title{
2D materials as semiconducting gate for field-effect transistors with inherent over-voltage protection and boosted ON-current
}

\author{
Qingkai Qian ${ }^{1}$, Jiacheng Lei ${ }^{1}$, Jin Wei (iD) ${ }^{1}$, Zhaofu Zhang ${ }^{1}$, Gaofei Tang ${ }^{1}$, Kailun Zhong ${ }^{1}$, Zheyang Zheng ${ }^{1}$ and Kevin J. Chen (iD)
}

Various 2D/3D heterostructures can be created by harnessing the advantages of both the layered two-dimensional semiconductors and bulk materials. A semiconducting gate field-effect transistor (SG-FET) structure based on 2D/3D heterostructures is proposed here. The SG-FET is demonstrated on an AIGaN/GaN high-electron mobility transistor (HEMT) by adopting single-layer MoS $_{2}$ as the gate electrode. The $\mathrm{MoS}_{2}$ semiconducting gate can effectively turn on and turn off the HEMT without sacrificing the subthreshold swing and breakdown voltage. Most importantly, the proposed semiconducting gate can deliver inherent over-voltage protection for field-effect transistors (FETs). Furthermore, the self-adjustable semiconducting gate potential with drain bias can even boost the ON-current while guaranteeing the safe operation of FET. In implementing the semiconducting gate, the layered two-dimensional materials such as the adopted $\mathrm{MoS}_{2}$ have several important benefits such as the feasibility of high-quality crystals on different gate dielectrics and the good controllability of semiconducting gate depletion threshold voltage by the layer thickness. The demonstrated semiconducting gate as over-voltage protection for HEMT can be extended to other FETs, which can become another advantageous arena for the possible applications of the layered two-dimensional materials.

npj 2D Materials and Applications (2019)3:24; https://doi.org/10.1038/s41699-019-0106-6

\section{INTRODUCTION}

Field-effect transistor (FET), as a voltage-driven device with large input impedance, is at the heart of modern semiconductor technologies (e.g., CMOS, TFT, compound semiconductor HEMT, etc.) supporting a wide range of existing and emerging applications. ${ }^{1-7}$ These applications include low-power FETs in logic and analog IC's for high-speed computing and loT, ${ }^{4,5}$ HEMTs/ MISFETs based on compound semiconductors (e.g., GaN, SiC) for high-power and high-frequency switchings. ${ }^{6,7}$ Despite the above advantages and broad applications, the voltage-driven FETs have a drawback of being very susceptible to the overloaded gate voltage. A large over-voltage gate stress can easily result in severe threshold voltage instabilities ${ }^{8-11}$ or even lead to long-term degradation (e.g., breakdown) of the gate dielectric or semiconductor barrier layer between the gate and the channel. ${ }^{12-14}$ Although power FETs are designed to sustain large drain bias, they are equally vulnerable to the forward gate overstress. Various gate over-voltage protection techniques have long been developed for FETs. ${ }^{15-17}$ These protection schemes can be categorized into two types: current limiting and voltage limiting. ${ }^{15}$ However, all these solutions require external peripheral circuits or components such as the bootstrapped FETs, Zener diode, etc, which not only could lead to higher cost and increased parasitics, but also impose extra difficulties for monolithic integration.

A voltage-clamping scheme, when inherently embedded into the gate electrode, would provide internal over-voltage protection without taking up any additional device areas. Such a solution has been lacking up to now, but becomes feasible with the emergence of two-dimensional semiconductor materials which can be conveniently integrated with the bulk materials. By using $2 \mathrm{D}$ semiconductors as the gate materials above the FET channel, an inherent over-voltage protection can be provided. The proposed two-dimensional semiconducting gate field-effect transistor (SG-FET) structure features a thin layer of moderately doped semiconductor as the gate, instead of the conventional conducting gate (CG) based on metal or heavily doped polysilicon. $^{2-5,18}$ As a result, the conductivity of SG can be effectively modulated by the gate electric field. The SG should have the same type of carrier as that of the active channel. For the case of an n-channel FET, a positive gate bias applied to the n-type SG would tend to partially deplete the SG. If the doping concentration of the SG is carefully tuned so that it becomes completely depleted when the underlying channel is fully turned on, any additional voltage appeared at the gate terminal would be decoupled from the gate dielectric (or semiconductor barrier) and the underlying FET channel, resulting in an inherent gate overvoltage protection.

In this work, the SG-FET with inherent gate over-voltage protection is demonstrated by adopting single-layer $\mathrm{MoS}_{2}$ as the SG for an AIGaN/GaN HEMT. The layered two-dimensional semiconductors such as $\mathrm{MoS}_{2}$ are especially attractive and suitable as SG because they present a dangling-bond-free surface and well-controlled atomically-thin layer thickness. ${ }^{1,19-21}$ Furthermore, the layered two-dimensional semiconductors can be deposited or transferred to the gate region during device processing rather easily. ${ }^{21-23}$ The demonstrated $\mathrm{MoS}_{2}$ SG-HEMT can maintain excellent turn-on characteristics such as a subthreshold swing (SS) of $63 \mathrm{mV} / \mathrm{dec}$, ON-current of $460 \mathrm{~mA} / \mathrm{mm}$, and a breakdown voltage of $408 \mathrm{~V}$ for HEMT with a gate-to-drain distance of $5 \mu \mathrm{m}$. Most importantly, without using any insulating gate dielectric, the SG-HEMT can at least sustain gate bias as high as $20 \mathrm{~V}$ and at the same time maintain a significantly suppressed gate leakage and boost the ON-current.

\footnotetext{
${ }^{1}$ Department of Electronic and Computer Engineering, Hong Kong University of Science and Technology, Clear Water Bay, Hong Kong SAR, China Correspondence: Qingkai Qian (qqian@connect.ust.hk) or Kevin J. Chen (eekjchen@ust.hk)
}

Received: 9 February 2019 Accepted: 22 May 2019

Published online: 13 June 2019 


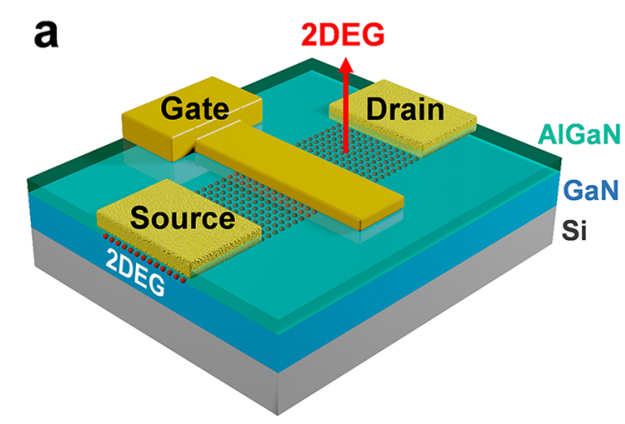

C

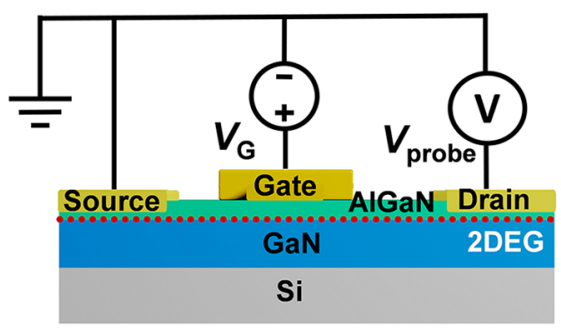

b

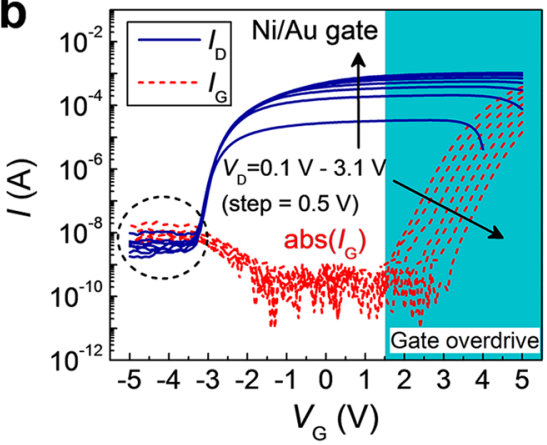

d

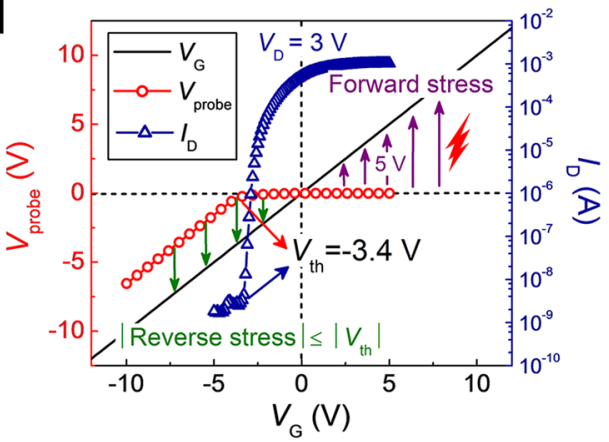

Fig. 1 Gate over-voltage and leakage issue of AIGaN/GaN HEMT with Schottky metal gate (MG). a Schematic drawing of a conventional AlGaN/GaN HEMT with Schottky Ni/Au metal gate. b Transfer curves and gate leakages of the MG-HEMT. c Probe setup to detect the channel potential at different gate biases. $\mathbf{d}$ Measured channel potential (red open circles) and the effective gate stresses (purple and olive arrows). The large forward gate stress without any protection can induce severe device degradations

\section{RESULTS}

Over-voltage issue of conventional MG-HEMT

The safe operation of conventional HEMT with a Schottky metal gate (MG) relies critically on the gate overstress protections. ${ }^{17}$ Figure 1a schematically draws the device structure of a conventional MG-HEMT. Two-dimensional electron gas (2DEG) is formed at the $\mathrm{AlGaN} / \mathrm{GaN}$ heterojunction interface, due to the spontaneous polarizations of $\mathrm{AlGaN}$ and $\mathrm{GaN} .{ }^{24}$ Source and drain ohmic contacts to 2DEG are formed by metal deposition and high-temperature annealing. The active channel regions are defined by ion implantation. The MG is formed with $5 \mathrm{~nm} / 6 \mathrm{~nm}$ $\mathrm{Ni} / \mathrm{Au}$. The detailed fabrication process can be found in the "Methods" section. Figure 1b shows the performance of the fabricated MG-HEMT. The device shows depletion-mode (Dmode) characteristics, because of the polarization-induced high carrier density of 2DEG under the gate. Relatively small ON/OFF ratio $\left(10^{5}\right)$ is obtained, due to the large reverse gate leakage. The gate leakage becomes especially large at forward gate bias, which can become comparable to the drain current and even induce a negative $I_{D}$. Not only can this large gate leakage cause a poor isolation between the gate control and channel current flow, it is also further responsible for the threshold voltage instability ${ }^{9,17}$ and the long-term degradation of the device performance (Supplementary Fig. 1).

The effective gate stress on the AIGaN barrier can be detected by measuring the voltage difference between the $M G$ and the 2DEG channel. The measurement setup is schematically drawn in Fig. 1c, in which the drain electrode is used as a probe to sense the channel potential. The $V_{\mathrm{G}}$-dependent channel potential is measured and plotted in Fig. 1d, with the effective gate stresses (i.e., voltage difference between the MG and 2DEG channel) indicated by the arrows. Owing to the depletion of 2DEG channel under the gate, the reverse gate stress on the AIGaN barrier is limited to the depletion threshold voltage of the 2DEG, which just explains the reverse gate leakage saturation in Fig. $1 \mathrm{~b}$. In contrast to the limited reverse gate stress, the forward gate stress is forced freely onto the whole AIGaN barrier without any protection, as indicated by the purple arrows in Fig. 1d. The unrestrained forward gate stress of MG-HEMT is responsible for the observed large gate leakages and the severe device performance degradations.

\section{Fabrication and characterization of SG-HEMT}

Instead of using the MG, the adoption of a semiconducting gate above the AlGaN barrier can provide a forward over-voltage protection and a suppressed gate leakage for AIGaN/GaN HEMT. The semiconducting gate should be able to be depleted by the large forward gate bias. In other words, the semiconducting gate should have the same type of carriers as the channel (n-type in our case), and more importantly it should be thin enough and moderately doped, thus its conductivity can be effectively modulated by the gate electric field. However, these requirements have imposed severe challenges to the use of conventional bulk semiconductors as the semiconducting gate. It is difficult to deposit or integrate thin film of bulk semiconductors on the varied gate dielectrics (e.g., the often-used amorphous high-k dielectrics for MOSFET) with high crystal quality. Even though $n-G a N$ can be epitaxially grown on the AIGaN barrier, it is still quite a challenge to maintain a small $n-G a N$ thickness and at the same time suppress the surface scatterings caused by the surface dangling bonds and the high-concentration dopants (to compensate the negative interface spontaneous polarization charges). In contrast to the conventional bulk semiconductors, the layered twodimensional semiconductors, such as $\mathrm{MoS}_{2}$ and $\mathrm{WSe}_{2}$ from the transition metal dichalcogenide (TMD) family, have a danglingbond free surface. ${ }^{19-21,25,26}$ As a result, they can maintain a high carrier mobility even with an atomically-thin layer thickness, which can even outperform that of SOI (silicon-on-insulator) and makes them competitive as channel materials for the next generation transistors. $^{27-29}$ Besides, these layered two-dimensional 


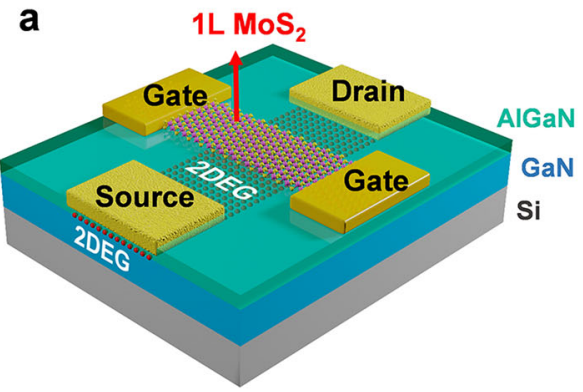

b

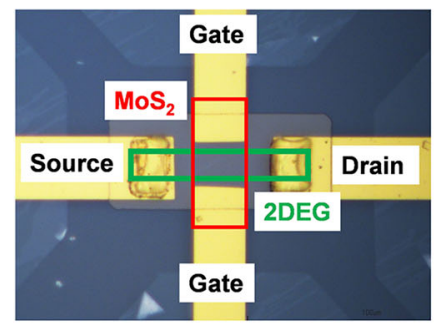

C

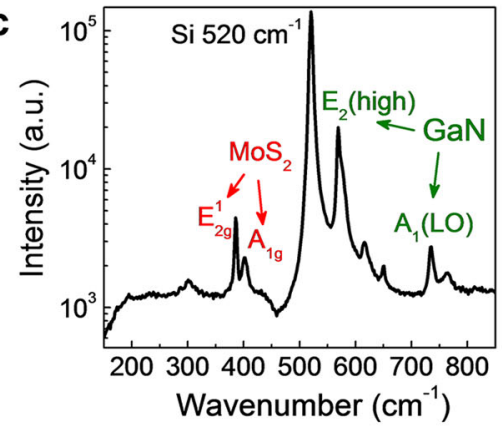

d

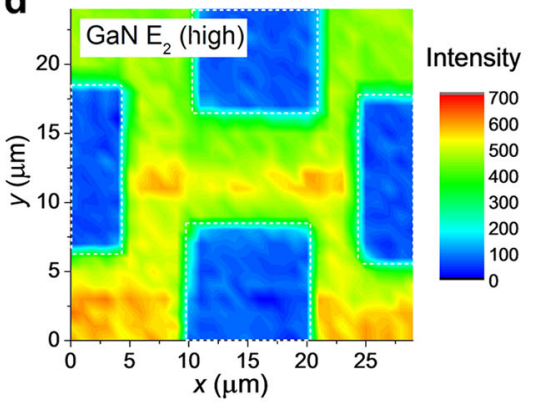

g

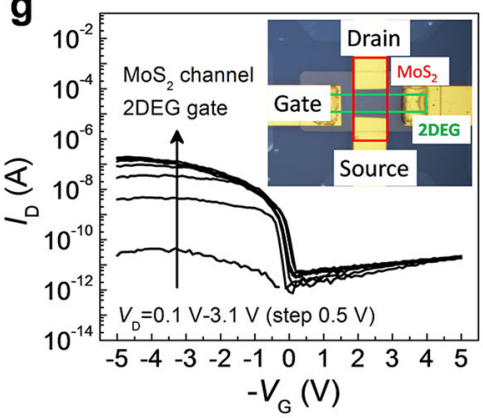

e

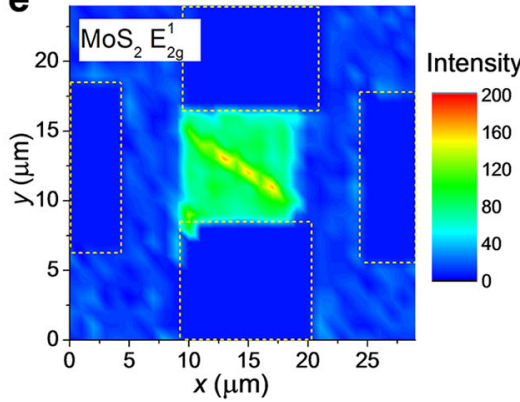

h

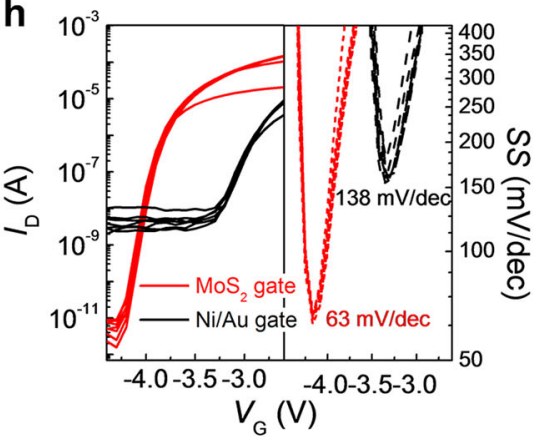

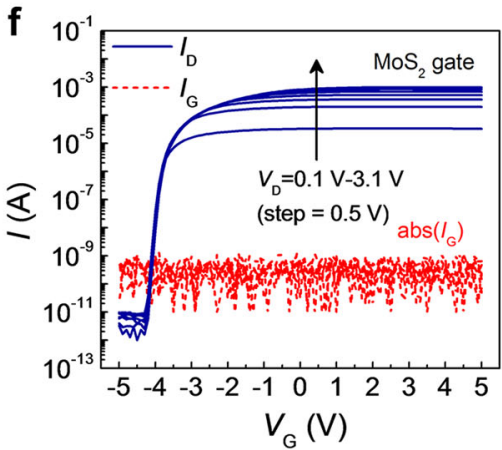

i

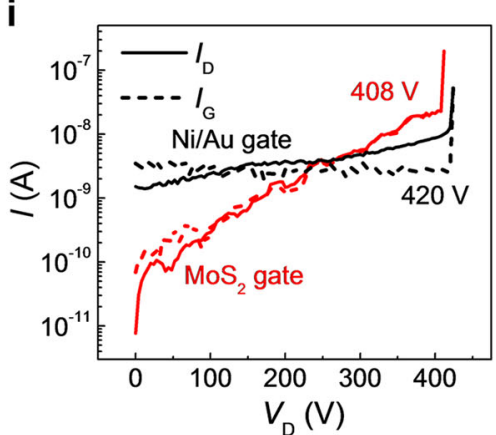

Fig. 2 AIGaN/GaN HEMT with single-layer $\mathrm{MoS}_{2}$ as a semiconducting gate (SG). a Schematic drawing of AIGaN/GaN HEMT with single-layer $\mathrm{MoS}_{2}$ as semiconducting gate. MoS 2 is exposed to air. b Optical microscope image of a fabricated SG-HEMT. c Raman spectrum of single-layer $\mathrm{MoS}_{2}$ on $\mathrm{AlGaN} / \mathrm{GaN}$ heterostructure. Raman intensity mapping of $\mathbf{d} \mathrm{GaN} E_{2}$ (high) peak and e $\mathrm{MoS}_{2} E_{2 g}^{1}$ peak. The dotted areas correspond to the metal electrodes. $\mathbf{f}$ Transfer curves of SG-HEMT and the corresponding gate leakages. $\mathbf{g}$ Transfer curves of the same device measured as a $\mathrm{MoS}_{2}$ transistor (see inset), noting that the horizontal axis $V_{\mathrm{G}}$ is inverted. $\mathrm{MoS}_{2}$ is depleted when the gate of SG-HEMT is largely forward biased. h Transfer curves, subthreshold swing (SS), and $\mathbf{i}$ off-state breakdown of MG-HEMT and SG-HEMT

semiconductors can be grown on and easily transferred to various substrates. ${ }^{21,23,30,31}$ All these properties have lent the layered twodimensional semiconductors a special advantage as a suitable candidate for the implementation of the semiconducting gate.

Experimentally, $\mathrm{MoS}_{2}$ is widely reported with $\mathrm{n}$-type conductivity, ${ }^{1,2,23,32}$ while $\mathrm{WSe}_{2}$ is more frequently reported with p-type or ambipolar conductivity. ${ }^{2,22,33}$ The n-type $\mathrm{MoS}_{2}$ is adopted here as a semiconducting gate for AIGaN/GaN HEMT to demonstrate the gate over-voltage protection capability. Figure 2 a schematically shows the device structure of the SG-HEMT. The almost-continuous $\mathrm{MoS}_{2}$ film is grown by the chemical vapor deposition (CVD) method on sapphire substrate and then transferred onto the $\mathrm{AlGaN} / \mathrm{GaN}$ sample. Ti/Au $(10 \mathrm{~nm} / 100 \mathrm{~nm})$ stack is deposited on $\mathrm{MoS}_{2}$ outside the active 2DEG channel as the gate electrode pads. Two gate pads are fabricated, which allow us to measure the same device as a $\mathrm{MoS}_{2}$ transistor to monitor the $\mathrm{MoS}_{2}$ conductivity. The detailed device fabrication processes can be found in the "Methods" section. The microscopic image of the fabricated SG-HEMT is shown in Fig. $2 \mathrm{~b}$. Due to the relatively low substrate contrast, single-layer $\mathrm{MoS}_{2}$ is almost invisible under the microscope. The successful transfer of $\mathrm{MoS}_{2}$ thin film onto the $\mathrm{AlGaN} / \mathrm{GaN}$ heterostructure is verified by the Raman spectrum in Fig. 2c. Two strong Raman peaks of $\operatorname{MoS}_{2}\left(E_{2 g}^{1}\right.$ and $\left.A_{1 g}\right)$ are clearly observed, and the peak distance of $19 \mathrm{~cm}^{-1}$ suggests that the $\mathrm{MoS}_{2}$ film is single-layer. ${ }^{26}$ The Raman intensity mappings of GaN $E_{2}$ (high) and $\mathrm{MoS}_{2} E_{2 g}^{1}$ peaks are shown in Fig. 2d, e respectively. The blue areas in Fig. $2 \mathrm{~d}$ correspond to the metal electrodes, and the shape of the $\mathrm{MoS}_{2}$ SG above the AIGaN barrier can be clearly identified by the high-intensity green area in Fig. 2e.

Transfer curves and gate leakages of the AIGaN/GaN HEMT with $\mathrm{MoS}_{2}$ semiconducting gate are measured and plotted in Fig. $2 \mathrm{f}$. The device shows almost no hysteresis, since it is mainly determined by the AIGaN/GaN interface. The SG can switch the 2DEG channel current effectively. Compared with the MG-HEMT in Fig. $1 \mathrm{~b}$, a slightly more negative threshold voltage is observed and can be attributed to the relatively smaller work function of $\mathrm{MoS}_{2}$ than $\mathrm{Ni}^{34}$ Most importantly, in contrast to the MG-HEMT, the SGHEMT exhibits a much smaller gate leakage. For example, the gate leakage is at least suppressed by five orders of magnitude at $5 \mathrm{~V}$, which is still limited by the equipment resolution of the gate probe. The fabricated SG-HEMT in Fig. $2 \mathrm{~b}$ can also be measured as 
a $\mathrm{MoS}_{2}$ transistor. The 2DEG channel is used as the back gate, while the two gate pads are used as the source and drain electrodes instead, as depicted in the inset of Fig. $2 \mathrm{~g}$. The transfer curves of the $\mathrm{MoS}_{2}$ transistor are plotted in Fig. 2g, with the gate voltage already being inverted (i.e., $-V_{\mathrm{G}}$ ). When measuring the SGHEMT with a gate bias of $V_{G}$, from the $M_{2} S_{2}$ transistor point of view, the $2 \mathrm{DEG}$ gate voltage is just $-V_{\mathrm{G}}$. So, the inverted $V_{\mathrm{G}}$ in Fig. $2 \mathrm{~g}$ can facilitate the identification of the conductivities of both $\mathrm{MoS}_{2}$ and 2DEG, through a quick comparison between Fig. 2f, g. It becomes clear that the $M_{0} S_{2}$ film can be depleted when the $\mathrm{MoS}_{2}$-to-2DEG voltage (i.e., $V_{\mathrm{G}}$ in Fig. $2 \mathrm{f}$ ) exceeds $0 \mathrm{~V}$, when the 2DEG channel (with a threshold voltage of $-4 \mathrm{~V}$ ) has already been sufficiently turned on. The $\mathrm{MoS}_{2}$ SG maintains or even has better conductivity during the turn-off of the 2DEG channel, with the Fermi level of $\mathrm{MoS}_{2}$ moving upward only slightly due to the increased electron density. As a result, even though as a semiconductor with tunable conductivity for $\mathrm{MoS}_{2}$, its capacitive coupling to 2DEG will not be weakened during the device switching off. A high ON/OFF ratio of $10^{9}$ is observed, owing to the small gate leakage. For the same reason, a SS as low as $63 \mathrm{mV} / \mathrm{dec}$ is achieved (Fig. 2h). Moreover, the SG imposes no penalty on the breakdown voltage, as shown in Fig. $2 \mathrm{i}$.

\section{SG-HEMT with varied SG carrier densities}

The performance of SG-HEMT in Fig. 2 is measured with singlelayer $\mathrm{MoS}_{2}$ exposed to air. It is known that when exposed to air, both the doping concentration and the carrier mobility of $\mathrm{MoS}_{2}$ can be significantly influenced by the air adsorptions. ${ }^{32,35,36}$ To alleviate the adsorption influence and enhance the stability of the device performance, the SG-HEMT is further passivated by ALD of 5-nm $\mathrm{ZrO}_{2}$ and $15-\mathrm{nm} \mathrm{Al}_{2} \mathrm{O}_{3}$, as schematically drawn in Fig. 3a. Before the high-k deposition, 20-min remote $\mathrm{N}_{2}$ plasma treatment is used as a surface functionalization technique to promote the uniform dielectric depositions. ${ }^{37,38} \mathrm{ZrO}_{2}$ has a larger dielectric constant, which is beneficial for the carrier mobility enhancement of $\mathrm{MoS}_{2}$ through impurity charge screening, ${ }^{39,40}$ while $\mathrm{Al}_{2} \mathrm{O}_{3}$ is used to achieve better isolation capability and to further stabilize the device performance. ${ }^{41}$

Transfer curves of the SG-HEMT after high-k dielectric passivation are plotted in Fig. $3 \mathrm{~b}$. The device is measured with a relatively larger gate swing (from $-10 \mathrm{~V}$ to $10 \mathrm{~V}$ ). No obvious threshold voltage changes are observed after the dielectric passivation. Compared with the SG-HEMT before passivation (Fig. 2f), the gate leakage increases. This gate leakage increase can be attributed to the changes of doping conditions in $\mathrm{MoS}_{2} \mathrm{SG}$, as indicated by the transfer curves of $\mathrm{MoS}_{2}$ transistor in Fig. $3 \mathrm{~b}$ (black solid lines). After dielectric passivation, the conductivity of $\mathrm{MoS}_{2}$ SG increases significantly as a result of the increased carrier density (as revealed by the threshold voltage shift) and the boosted electron mobility (from 0.06 to $6 \mathrm{~cm}^{2} / \mathrm{V} \mathrm{s}$ ). Nevertheless, the gate leakage of SGHEMT is still much smaller than that of MG-HEMT (Fig. 1b). Moreover, a saturation of the forward gate leakage is observed in Fig. 3b, allowing a much larger gate swing for SG-HEMT (at least $-20 \mathrm{~V}$ to $20 \mathrm{~V}$, see Supplementary Fig. 2) compared with the MGHEMT ( 6 V at most at forward gate bias, see Supplementary Fig. 1). The off-state current is mainly contributed by the gate leakage. Compared with SG-HEMT before dielectric passivation, the minimum gate leakage increases slightly, possibly due to the increased electron density and the reduced work function of $\mathrm{MoS}_{2}$ SG after passivation. Besides the suppressed gate leakage and large gate swing, the pulsed $I-V$ characteristics of SG-HEMT suggests that SG-HEMT can at least respond to $5-\mu$ s fast switching (Supplementary Fig. 3). From the transfer curves in Fig. 3b, the minimum resistances of both $\mathrm{MoS}_{2} \mathrm{SG}$ and 2DEG channel can be estimated to be $R_{\mathrm{SG}}=131 \mathrm{k} \Omega$ and $R_{2 \mathrm{DEG}}=2.5 \mathrm{k} \Omega$, then the delay time can be roughly estimated by $\left(R_{\mathrm{SG}}+R_{2 \mathrm{DEG}}\right) C_{\mathrm{AlGaN}}=22 \mathrm{~ns}$, in which $C_{\mathrm{AlGaN}}=0.17 \mathrm{pF}$ is the capacitance of AlGaN barrier. It becomes clear that the main bottleneck of the switching speed comes from the low carrier mobility $\left(6 \mathrm{~cm}^{2} / \mathrm{N}\right)$ and the large
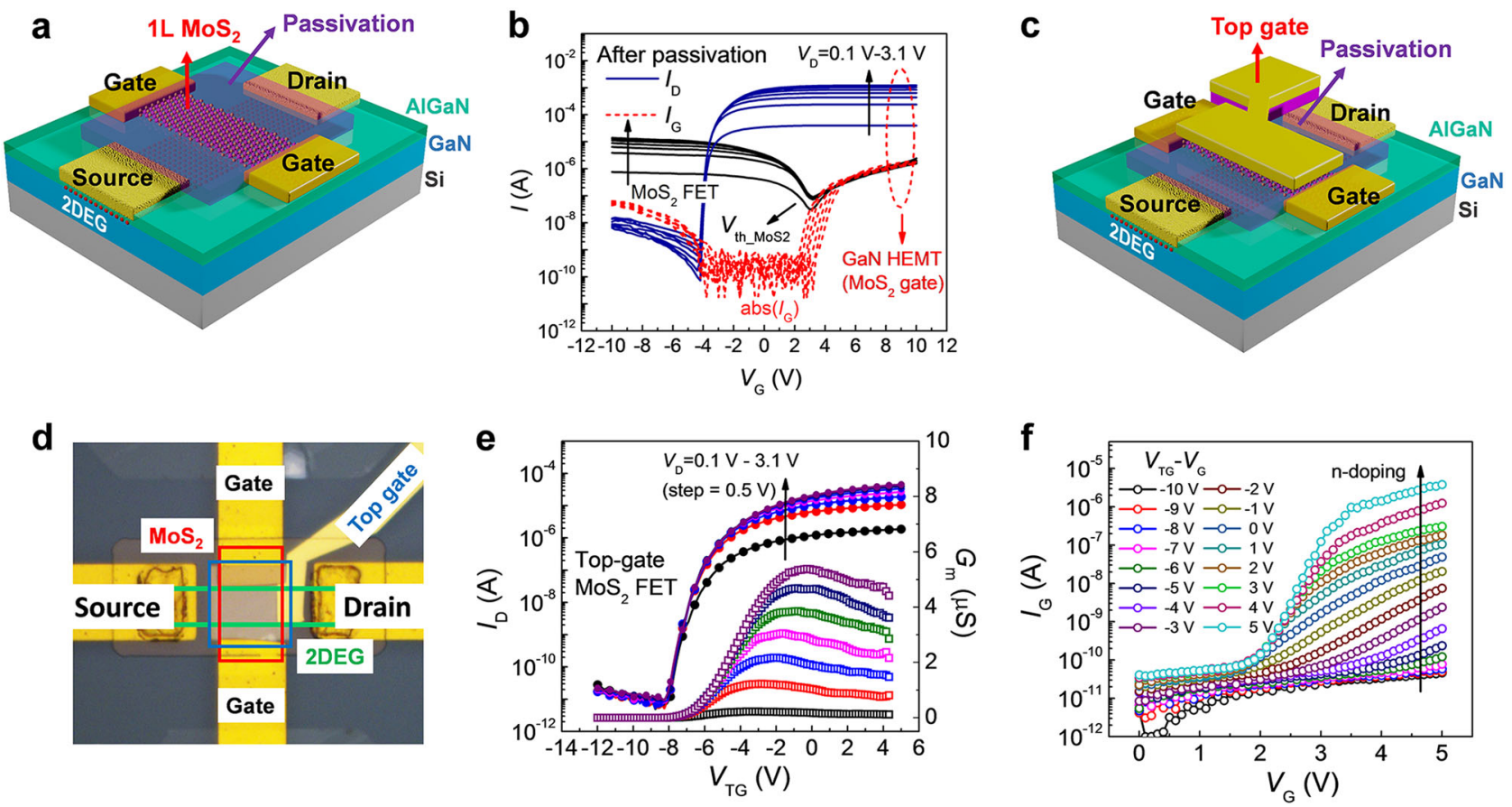

Fig. 3 Dielectric passivated SG-HEMT and its gate leakage dependence on the SG carrier density. a Schematic illustration of MoS 2 SG-HEMT passivated by $5-\mathrm{nm} \mathrm{ZrO}_{2}$ and $15-\mathrm{nm} \mathrm{Al}_{2} \mathrm{O}_{3}$. The $\mathrm{ZrO}_{2}$ and $\mathrm{Al}_{2} \mathrm{O}_{3}$ layers are deposited by ALD to isolate the MoS from the air. $\mathbf{b}$ Transfer curves and gate leakages of SG-HEMT after high-k dielectric passivation. Transfer curves of the $\mathrm{MoS}_{2}$ transistor are also plotted as the black solid lines, with the 2DEG back-gate voltage already being inverted. c Schematic illustration of $\mathrm{MoS}_{2}$ SG-HEMT with an additional top gate to control the doping of $\mathrm{MoS}_{2}$ and $\mathbf{d}$ the corresponding optical image. e Transfer curves of the top-gate $\mathrm{MoS}_{2}$ transistor. $\mathbf{f}$ Forward gate leakages of SG-HEMT with different electric-field modulated SG carrier densities 
contact resistance $(100 \Omega \mathrm{mm})$ of single-layer $\mathrm{MoS}_{2}{ }^{42}$ both of which could be further optimized in the future, e.g., by adopting multilayer $\mathrm{MoS}_{2}{ }^{31,42,43}$ or other high-mobility two-dimensional semiconductors $22,44,45$ as the SG, or using SG only at the channel edge (Supplementary Fig. 3).

The comparison experiments of SG-HEMT before and after high-k dielectric passivation suggest that the gate leakage can be significantly influenced by the doping conditions of the SG part of the device. The dielectric passivation affects the carrier density of SG mainly by changing the surface adsorptions in air, which is relatively hard to control in practice. On the contrary, the carrier density of SG can be conveniently and monotonously tuned by an external electric field. To further study the SG doping influence over the gate leakage and the device performance, a top gate is added to SG-HEMT. The schematic illustration of a fabricated device and the corresponding optical image are shown in Fig. $3 c, d$ respectively. As demonstrated by the transfer curves in Fig. $3 e$, the SG carrier density can be effectively modulated by the top gate. The top-gate $\mathrm{MoS}_{2}$ transistor has a more negative threshold voltage than $\mathrm{MoS}_{2}$ transistor with a 2DEG back-gate, which could be related to the better air isolation capability of the top-gate SGHEMT with the additional top-gate metal. The gate leakages of SGHEMT are measured again, with SG carrier density tuned by the relative voltage difference between the top gate and the $\mathrm{MoS}_{2} \mathrm{SG}$. The gate leakage depends strongly on the doping concentration of $\mathrm{SG}$, as shown in Fig. 3f. Consistent with the previously observed trend for SG-HEMT before and after high-k passivation, SG with a higher doping concentration is more difficult to be depleted by the forward gate bias, leading to a larger gate stress on the AIGaN barrier and a larger forward gate leakage.
Clamped forward gate stress and boosted ON-current

Because of the adoption of SG, the gate leakage of SG-HEMT can be successfully suppressed, without sacrificing the SS and the breakdown voltage. The large forward gate bias not only turns on the 2DEG channel, but also depletes the SG and increases the gate resistance exponentially when $V_{G}$ approaches the depletion threshold voltage of the SG. All the evidence suggest that the semiconducting nature of $\mathrm{MoS}_{2}$ is the key to the improved SGHEMT performance and the doping concentration of SG is an important design parameter. To quantitatively analyze the gate leakages of SG-HEMT, a simplified one-dimensional model is proposed in Fig. 4a. In this model, the 2DEG and the $\mathrm{MoS}_{2}$ SG are simplified to be electrically contacted on the same side. The current density of vertical gate leakage is assumed to depend only on the vertical potential difference. The vertical gate leakage current accumulates and flows horizontally along 2DEG and $\mathrm{MoS}_{2}$, which further influences the vertical voltage differences, after considering the mutual conductivity modulations of the 2DEG channel and the $\mathrm{MoS}_{2}$ SG by the vertical potential difference. During the calculation, the mutual conductivity modulations are determined by the transfer characteristics in Fig. 3b, while the parameters for the vertical gate leakage are extracted from the exponentially increased gate leakage of MG-HEMT in Fig. 1b. A detailed derivation of the gate leakage can be found in the Supplementary Information.

Based on the simplified one-dimensional model, the potential distribution along the gate width can be analytically calculated, in which the depletion threshold voltage of $\mathrm{MoS}_{2}$ by the 2DEG back gate is assumed to be $2 \mathrm{~V}$. The calculation results in Fig. $4 \mathrm{~b}$ clearly suggest that the potential of the SG above the 2DEG channel is clamped close to the depletion voltage of the SG, with the extra

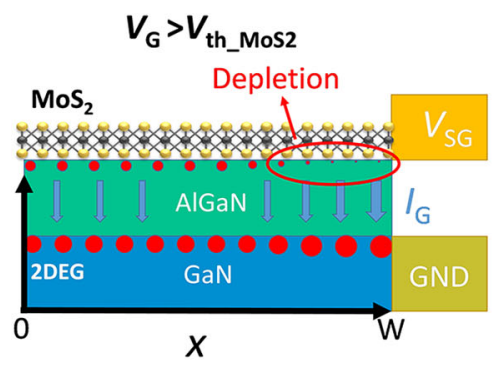

b
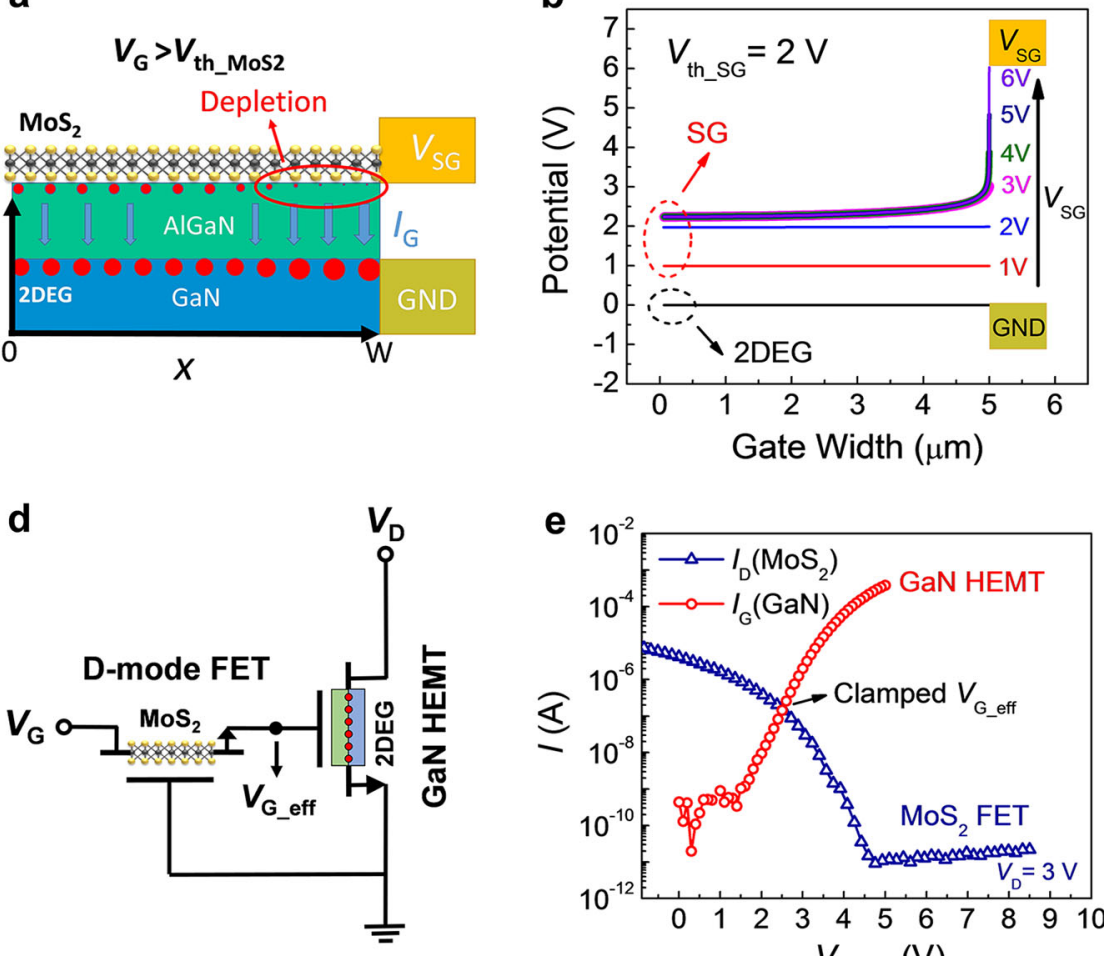

e

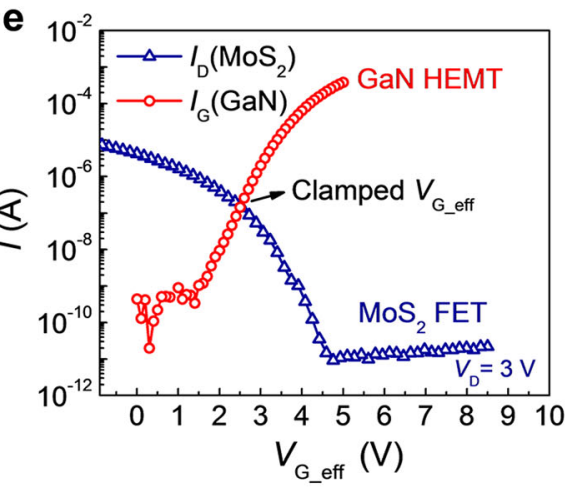

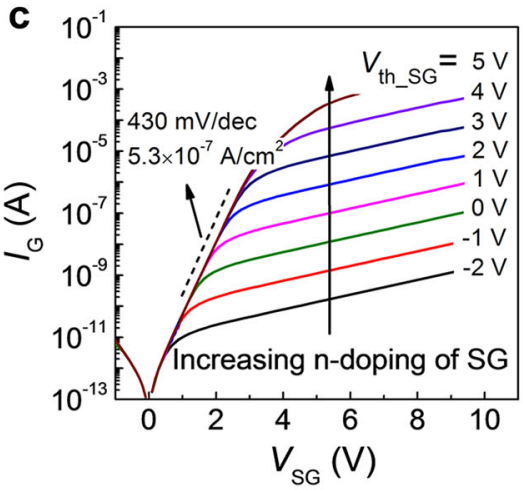

f

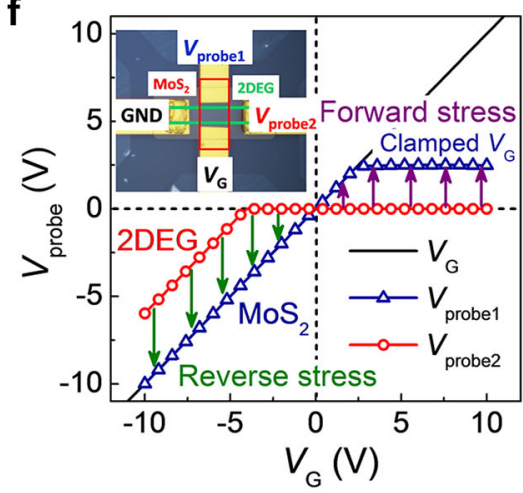

Fig. 4 Gate voltage clamping of SG-HEMT. a Schematic of a simplified one-dimensional model to analytically calculate the gate leakage of SGHEMT. MoS 2 is depleted when the SG is largely forward biased. b Calculated potential distribution along the width of SG. c Calculated gate

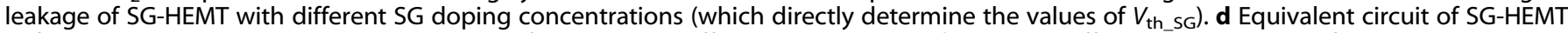
at forward gate bias and e the determination of the clamped effective gate voltage. $\mathbf{f}$ Measured effective gate voltage of MoS $_{2}$ SG with probe setup shown in the inset. For $V_{G}<V_{\text {th_Mos2 }}$, the gate voltage is applied on the whole SG. While for $V_{G}>V_{\text {th_Mos2 }}$, the effective gate voltage is clamped to $V_{\text {th_Mos2 }}$. As a result, SG can deliver an inherent forward gate over-voltage protection 
gate voltage mainly being sustained by the SG region at the channel edge. The depletion and voltage clamping of the SG are also verified by a 2D simulation (Supplementary Fig. 4). The gate leakages of SG-HEMT with different SG doping concentrations (which yield different depletion threshold voltages $V_{\text {th_sG }}$ ) are also calculated and shown in Fig. 4c. SG with higher doping concentration clamps the effective gate voltage to a higher value, resulting in a larger gate leakage. The gate leakage shows a saturation behavior when the SG becomes depleted, which is consistent with the experimental results in Fig. $3 b, f$ (noting that the gate leakage in Fig. $2 f$ is still limited by the equipment resolution of the gate probe). The saturation behavior of the forward gate leakage due to the depletion of SG is not as sharp as that of the reverse gate leakage saturation in Fig. 1b. Our theoretical calculations suggest that this is caused by the relatively poor SS of SG conductivity modulation by the 2DEG back gate in our experiment (Supplementary Fig. 5).

The operating principle of using SG to clamp the forward gate voltage and to suppress the gate leakage can be further explained by an equivalent circuit drawn in Fig. $4 \mathrm{~d}$. In this circuit, the SG is replaced by a D-mode $\mathrm{MoS}_{2}$ transistor, which is serially connected to the gate of the MG-HEMT. The gate of the $\mathrm{MoS}_{2}$ transistor is connected to the source of the GaN HEMT. The gate voltage of HEMT is applied through the semiconducting $\mathrm{MoS}_{2}$ and a large forward $V_{G}$ can deplete the $\mathrm{MoS}_{2}$, resembling the case in SGHEMT. For sufficiently large $V_{G}$, the current flowing through the D-mode $\mathrm{MoS}_{2}$ transistor is not very sensitive to $V_{\mathrm{G}}$, because the device is in the subthreshold region. As a result, the effective gate voltage $\left(V_{\mathrm{G} \text { eff }}\right)$ can be quantitatively determined by the cross point of the $\mathrm{MoS}_{2}$ channel current and the HEMT gate leakage current, as shown in Fig. 4e. Owing to the depletion of the D-mode $\mathrm{MoS}_{2}$ transistor, the effective gate voltage of HEMT is clamped. In contrast to the over-voltage protection by bootstrapped $\mathrm{FET}^{17}$ the proposed SG principally has no limitation on the peak displacement current and thus the device switching speed, because it is a voltage-clamping technique, while the bootstrapped FET protection method only works for the FET with a large gate leakage current (e.g., metal-gate Schottky barrier HEMT). As a result, the proposed SG over-voltage protection can be implemented in all kinds of devices, such as the MIS-HEMT, regardless of the gate leakage (because the conductivity of semiconducting gate can be exponentially modulated and finally matches the gate leakage).

Experimentally, the clamping of the effective SG voltage can be verified by measuring the SG potential above the channel. The measurement setup is depicted in the inset of Fig. $4 \mathrm{f}$, and the measured SG potentials are plotted as the blue open triangles. For gate bias lower than the depletion threshold voltage of the SG, the gate voltage is effectively applied on the entire SG region. However, once the gate bias becomes larger than the depletion threshold voltage of the SG, the effective gate voltage is clamped. Besides the SG potential, the 2DEG channel potential is also plotted in Fig. $4 \mathrm{f}$ (red open circles). With the introduction of SG, both the forward and reverse gate stresses are now limited to the depletion threshold voltages of the SG and the 2DEG, respectively. Compared with the equivalent circuit, a semiconducting gate can provide an inherent over-voltage protection with a much more compact device structure. More importantly, the SG scheme inherently includes both forward and reverse over-voltage protections, owing to the depletion of both SG and 2DEG channel, which is absent in the equivalent circuit (at negative $V_{G}$ bias the D-mode $\mathrm{MoS}_{2}$ FET is vulnerable to gate breakdown).

The clamped effective gate voltage in Fig. $4 \mathrm{f}$ is measured with a floating drain $\left(I_{\mathrm{D}}=0 \mathrm{~A}\right)$. In practical applications, the drain is biased to deliver a drive current, which will change the vertical gate stress and influence the clamped gate voltage. Figure $5 a$ a

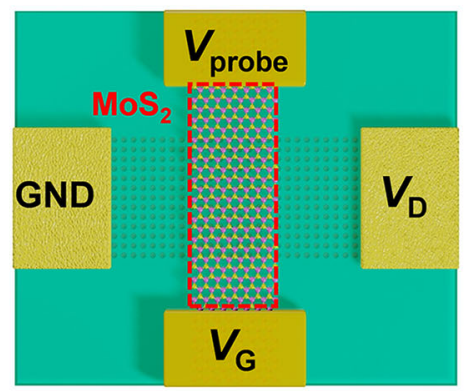

d

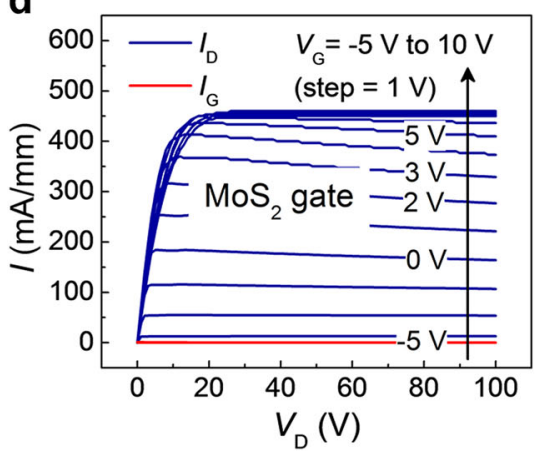

b

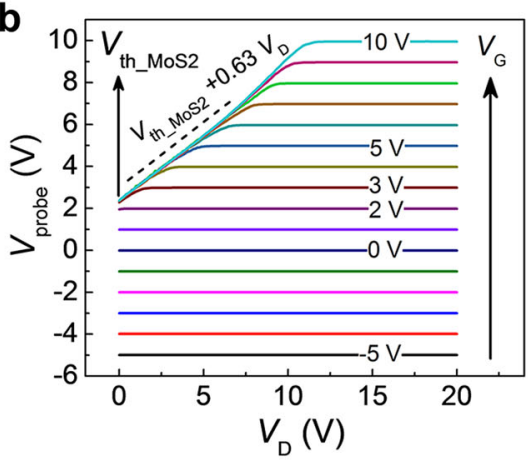

e

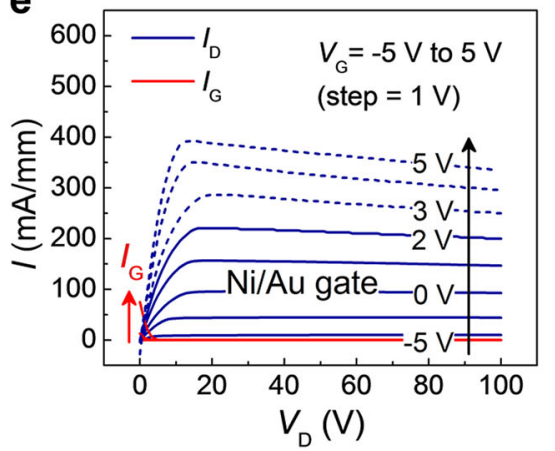

C
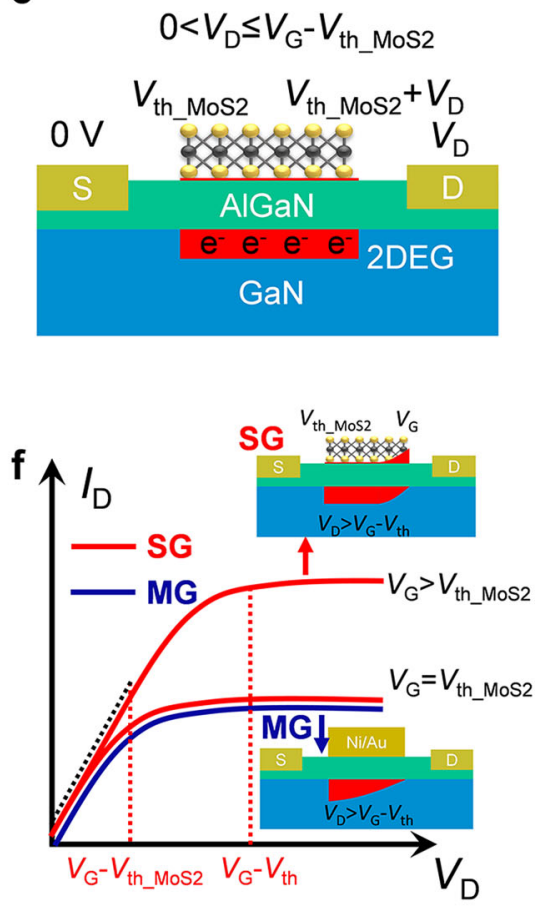

Fig. 5 Self-adjustable SG potential with drain biases and the safely boosted ON-current. a Top view of probe setup to measure the effective SG voltage of $\mathrm{MOS}_{2}$ SG-HEMT with a non-zero drain bias. b Measured probe voltage and its dependence on $V_{D}$ for a passivated MoS 2 SG-HEMT. c Cross-sectional schematic of SG potential distribution along the gate length. To guarantee a zero net current, the measured probe voltage is an average of the SG potential above the channel. Output curves of $\mathbf{d}$ MoS $_{2}$ SG-HEMT and e Ni/Au MG-HEMT. No sacrifice of the ON-current is observed for SG-HEMT. $\mathbf{f}$ Because of the self-adjustable gate potential distribution, while guaranteeing the safe operation, SG-HEMT can even have a higher average carrier density, a smaller channel depletion length and thus a larger saturation ON-current 
shows an experimental setup to detect the SG potential for different drain biases. The probe voltages with various gate and drain biases are measured and plotted in Fig. 5b. Consistent with the previous result, at $V_{\mathrm{D}}=0 \mathrm{~V}$, the measured SG voltage is clamped to the depletion threshold voltage of $\mathrm{MoS}_{2}$, i.e., $V_{\text {th }}$ Mos2 $=2.5 \mathrm{~V}$. With increased $V_{\mathrm{D}}$, the clamped probe voltage also increases, as shown by the dashed line in Fig. 5b. The line has a slope close to 0.5 , which can be explained by the SG potential distribution in Fig. 5c. Because of the significantly tuned SG conductivity and the strong capacitive coupling between $\mathrm{MoS}_{2}$ and 2DEG, the SG above the channel is not completely equipotential for a non-zero drain bias during the gate voltage clamping. For $V_{G}>V_{\text {th_Mos2 }}$, the depletion of SG near the source end clamps the SG potential to $V_{\text {th_Mos2. }}$. However, at the drain end, due to the positive drain bias $V_{D}$, the depletion of SG requires a higher SG potential. If the current flow inside the $\mathrm{MoS}_{2}$ layer is ignorable, the $\mathrm{MoS}_{2}$ layer can always be regarded as depleted and the drain-side $\mathrm{MoS}_{2}$ voltage should be $V_{\text {th_Mos2 }}+V_{\mathrm{D}}$. As long as the SG potential is still smaller than $V_{G}$, the vertical voltage difference between $\mathrm{MoS}_{2}$ SG and 2DEG will always be limited to the depletion threshold voltage of $\mathrm{MoS}_{2} \mathrm{SG}$. For a specific range of drain bias $\left(V_{D}<V_{G}-V_{\text {th }}\right.$ Mos2 2$)$, the carrier density of SG-HEMT is kept constant along the channel without any modulation, which is in sharp contrast to the carrier density reduction of MG-HEMT even for a small positive $V_{\mathrm{D}}$. Because the measured probe voltage is a weighted average of the SG potential to guarantee a zero net current, the clamped probe voltage increases with $V_{D}$ by a slope close to 0.5

The self-adjustable SG potential of SG-HEMT in Fig. 5c suggests that SG-HEMT can achieve a higher average carrier density and thus a larger ON-current than MG-HEMT, while maintaining the same maximum gate stress. Figure $5 \mathrm{~d}$, e shows the measured output characteristics of $\mathrm{MoS}_{2} \mathrm{SG}-\mathrm{HEMT}$ and Ni/Au MG-HEMT. The $\mathrm{ON}$-current drops at large drain bias due to self-heating effect. The $\mathrm{MoS}_{2}$ SG has a depletion threshold voltage of $2.5 \mathrm{~V}$ (i.e., $V_{\text {th_Mos2 }}=$ $2.5 \mathrm{~V}$ ), which limits the maximum vertical gate stress to $2.5 \mathrm{~V}$ and fixes the $R_{\mathrm{ON}}$ in linear region for $V_{\mathrm{G}}>V_{\mathrm{th}}$ Mos2. However, because of this self-adjustable voltage distribution of $\mathrm{SG}$, the output curves of SG-HEMT shows a longer linear region and an increased ONcurrent in saturation region for $V_{G}>V_{\text {th Mos2 }}$, which even outperforms that of the MG-HEMT. This safely-boosted ON-current of the SG-HEMT can be further explained by the different carrier density distributions of SG-HEMT and MG-HEMT. As schematically drawn in Fig. 5f, the output curves of SG-HEMT and MG-HEMT should have no big differences for $V_{\mathrm{G}}<V_{\text {th Mos2 }}$ (except for a possible threshold voltage difference due to different work function of $\mathrm{MoS}_{2}$ and $\mathrm{Ni} / \mathrm{Au}$ ). However, while maintaining the maximum gate stress and safe operation, SG-HEMT can be biased at $V_{\mathrm{G}}>V_{\text {th_Mos2, }}$ owing to the inherent gate over-voltage protection capability. Benefiting from the self-adjustable SG potential above the channel with increased drain biases, the SG-HEMT can have a higher average carrier density, as schematically shown by the insets of Fig. $5 f$. As a result, the ON-current is boosted for SG-HEMT while the safe operation of device is guaranteed. It is noticed that for very large $V_{\mathrm{G}}\left(V_{\mathrm{G}}=6-10 \mathrm{~V}\right)$, the ON-current in Fig. $5 \mathrm{~d}$ does not increase further, which indicates that the current flow inside the $\mathrm{MoS}_{2}$ layer itself may not be ignorable any more, and the drainside $\mathrm{MoS}_{2}$ voltage cannot be simply determined by $V_{\text {th MoS2 }}+V_{\mathrm{D}}$ (otherwise the $V_{D_{-} \text {sat }}$ and $I_{\mathrm{ON}}$ will continuously increase with $V_{\mathrm{G}}$ ). The drain-side $\mathrm{MoS}_{2}$ voltage can increase with $V_{\mathrm{D}}$ but with a smaller rate than 1 , then a specific large $V_{D}$ will be enough to deplete 2DEG at the drain side and the drain current will become saturated regardless of the large $V_{\mathrm{G}}$. After all, compared with MGHEMT with the same saturation current, the 2DEG density of SGHEMT will be more evenly distributed, owing to the self-adjustable SG potential distribution. As a result, the heat generation for SGHEMT will be less focused than MG-HEMT, which might explain the reduced self-heating effect of SG-HEMT in Fig. $5 d$, especially for large $V_{\mathrm{G}}$.

\section{DISCUSSION}

A semiconducting gate is proposed to provide inherent gate overvoltage protection for FETs. The semiconducting gate scheme is experimentally demonstrated on an AIGaN/GaN HEMT, by implementing single-layer $\mathrm{MoS}_{2}$ as the semiconducting gate. Dangling-bond-free 2D materials such as $\mathrm{MoS}_{2}$ are especially suitable for the implementation of SG-FET, as high-quality thin films could be transferred or deposited on various gate dielectrics and the threshold voltage of the SG can be conveniently controlled by the layer thickness. The demonstrated SG-HEMT shows no penalties to the ON/OFF switching, the current driving capability, and the breakdown voltage. Even though the switching speed can be influenced by the deteriorated conductivity of the semiconducting gate, this problem can be mitigated by further device structure optimization and engineering as mentioned in the main text. Most importantly, the SG can clamp the effective forward gate voltage to the depletion threshold voltage of the SG, which inherently sets a limit on the maximum allowed forward gate stress. Furthermore, because of the self-adjustable SG potential above the channel, the SG gate can even boost the ON-current, while guaranteeing the safe operation of SG-HEMT. The demonstrated AIGaN/GaN SG-HEMT has validated the feasibility and superiority of SG in providing over-voltage protection and boosting the ON-current, which can be further extended to other FETs to enhance the device reliability and stability in the future, and can find potential applications in cases where the over-voltage protection is critical or a boosted ONcurrent is demanded.

\section{METHODS}

$\mathrm{MoS}_{2}$ preparation

Almost-continuous single-layer $\mathrm{MoS}_{2}$ film is grown by CVD on a sapphire substrate. The $\mathrm{MoS}_{2}$ film is then transferred to the target substrate by a modified wet-transfer method. First, the sapphire substrate is spin coated by PMMA $950 \mathrm{~K} \mathrm{~A} 4$ at $3000 \mathrm{rpm}$, then soaked in $10 \% \mathrm{KOH}$ at $80^{\circ} \mathrm{C}$ for hours until PMMA separates from the sapphire substrate. The PMMA together with $\mathrm{MoS}_{2}$ is fished into deionized water (DIW) for several times. Before transferred to the target substrate, the PMMA is fished onto a temporal $\mathrm{SiO}_{2} / \mathrm{Si}$ substrate. After the PMMA dries naturally, adhesive tape is used to peel the PMMA together with $\mathrm{MoS}_{2}$ from the temporal substrate and to be pasted on the target substrate later. The $\mathrm{MoS}_{2}$ surface is cleaned by soaking in FHD-5 for $30 \mathrm{~min}$, and rinsed in DIW several times. The $\mathrm{MoS}_{2}$ is then dried by baking at $50^{\circ} \mathrm{C}$, which guarantees no contamination of chemicals or water layers on the $\mathrm{MoS}_{2}$ surface.

\section{Device fabrication}

The SG-HEMT is fabricated on an AIGaN/GaN heterostructure grown on a 4-in. (111) Si substrate. The epitaxial structure consists of a 4- $\mu \mathrm{m} \mathrm{GaN}$ buffer/transition layer and a 23.5-nm barrier layer (including 1.5-nm AIN, 20-nm AlGaN, and 2-nm GaN cap). The device fabrication commenced with the formation of source/drain ohmic contacts featuring $\mathrm{Ti} / \mathrm{Al} / \mathrm{Ni} / \mathrm{Au}$ stack annealed at $850^{\circ} \mathrm{C}$. Fluorine ion implantation is used to define the active regions and provide isolation between devices. Before transferring singlelayer $\mathrm{MoS}_{2}$ film to the sample, the GaN surface was cleaned by $\mathrm{O}_{2}$ plasma oxidation and $\mathrm{HCl}$ dipping. Then the $\mathrm{MoS}_{2} / \mathrm{PMMA}$ /tape stacks are pasted on the target AlGaN/GaN substrate and are further heated at $110^{\circ} \mathrm{C}$ for $4 \mathrm{~min}$ to promote the adhesion between $\mathrm{MoS}_{2}$ and the substrates. Both the tape and PMMA are then dissolved in acetone. Large-area $\mathrm{MoS}_{2}$ film (at least $5 \mathrm{~mm}$ ) can be stably transferred. The transferred $\mathrm{MoS}_{2}$ samples are further cleaned by sonication in acetone for at least $3 \mathrm{~h}$. MoS $\mathrm{M}_{2}$ is then etched by $\mathrm{SF}_{6}$ plasma into the designed pattern as the $\mathrm{SG}$. Ti/Au metal is deposited on $\mathrm{MoS}_{2}$ outside the active 2DEG channel as the gate pad. To passivate the device, $5-\mathrm{nm} \mathrm{ZrO}_{2}$ and $15-\mathrm{nm} \mathrm{Al}_{2} \mathrm{O}_{3}$ are deposited on top of the entire device by PEALD (Oxford Instruments OpAL ALD system), before 
which 20-min remote $\mathrm{N}_{2}$ plasma treatment is used as the surface functionalization. For device with an additional top gate, 5-nm Ni and 6$\mathrm{nm} \mathrm{Au}$ are deposited on top of $\mathrm{MoS}_{2}$ to electrically tune the carrier densities of $\mathrm{MoS}_{2}$. All the devices described in this work has a gate length $L_{g}$ of $10 \mu \mathrm{m}$, a gate-drain distance $L_{g d}$ of $5 \mu \mathrm{m}$, a gate-source distance $L_{g s}$ of $5 \mu \mathrm{m}$. HEMTs with Schottky metal $(5 \mathrm{~nm} / 6 \mathrm{~nm} \mathrm{Ni} / \mathrm{Au})$ gate were also fabricated for comparison.

\section{Characterizations}

The optical microscope images are taken by Nikon IC Inspection Microscope with Digital Camera (PHT-MIC1). The Raman spectrum is measured by Renishaw inVia confocal microscope in the backscattering geometry. $514 \mathrm{~nm} \mathrm{Ar}$ laser is used, with 100x objective. Notch filter of $100 \mathrm{~cm}^{-1}$ is equipped. The laser spot has a size of about $0.5 \mu \mathrm{m}$. The Raman mapping is conducted in a $30 \times 25$ array, each sampling has a relatively short signal accumulation time compared with a full scan of the Raman spectrum. The Raman background caused by metal electrodes is subtracted during mapping the Raman peak intensities. The electric device performances are measured by Agilent B1505A power device analyzer/ curve tracer inside a probe station. Several tens of devices are measured, all of which show very consistent device performances. To sense the effective gate/channel potential voltage, the corresponding probe is set to current mode with $I=0 \mathrm{~A}$.

\section{DATA AVAILABILITY}

The data that support the plots within this paper and other findings of this study are available from the corresponding author upon reasonable request.

\section{ACKNOWLEDGEMENTS}

The authors would like to thank all the supports from the technical staff in Nanoelectronics Fabrication Facility (NFF) and Material Characterization and Preparation Facility (MCPF) of The Hong Kong University of Science and Technology.

\section{AUTHOR CONTRIBUTIONS}

K.J.C. and Q.Q. conceived the experiments. Q.Q. and J.L. conducted the simulation. Q. Q. fabricated and characterized the devices with help from Z. Zhang, G.T., K.Z. and Z. Zheng. Q.Q. and J.W. discussed about the effective potential and Q.Q. conducted the measurement. Q.Q. and K.J.C. wrote the manuscript. All authors reviewed the manuscript.

\section{ADDITIONAL INFORMATION}

Supplementary Information accompanies the paper on the npj $2 D$ Materials and Applications website (https://doi.org/10.1038/s41699-019-0106-6).

Competing interests: The authors declare no competing interests.

Publisher's note: Springer Nature remains neutral with regard to jurisdictional claims in published maps and institutional affiliations.

\section{REFERENCES}

1. Radisavljevic, B., Radenovic, A., Brivio, J., Giacometti, V. \& Kis, A. Single-layer MoS 2 transistors. Nat. Nanotechnol. 6, 147-150 (2011).

2. Sachid, A. B. et al. Monolithic 3D CMOS using layered semiconductors. Adv. Mater. 28, 2547-2554 (2016).

3. Nomura, K. et al. Room-temperature fabrication of transparent flexible thin-film transistors using amorphous oxide semiconductors. Nature 432, 488-492 (2004).

4. Carter, R. et al. $22 \mathrm{~nm}$ FDSOI technology for emerging mobile, Internet-of-Things, and RF applications. IEDM Tech. Dig. 2016, 2.2.1-2.2.4 (2016).

5. Tang, J. et al. Flexible CMOS integrated circuits based on carbon nanotubes with sub-10 ns stage delays. Nat. Electron. 1, 191-196 (2018).

6. Huang, X., Liu, Z., Li, Q. \& Lee, F. C. Evaluation and application of 600 V GaN HEMT in cascode structure. IEEE Trans. Power Electron. 29, 2453-2461 (2014).

7. Hamada, K., Nagao, M., Ajioka, M. \& Kawai, F. SiC-emerging power device technology for next-generation electrically powered environmentally friendly vehicles. IEEE Trans. Electron Devices 62, 278-285 (2015).
8. Zafar, S. et al. A comparative study of NBTI and PBTI (charge trapping) in $\mathrm{SiO}_{2} /$ $\mathrm{HfO}_{2}$ stacks with FUSI, TiN, Re gates. VLSI Symp. Tech. Dig. 2006, $23-25$ (2006).

9. Lagger, P., Ostermaier, C., Pobegen, G. \& Pogany, D. Towards understanding the origin of threshold voltage instability of AIGaN/GaN MIS-HEMTs. IEDM Tech. Dig. 2012, 13.1.1-13.1.4 (2012).

10. Lee, J., Cho, I., Lee, J. \& Kwon, H. Bias-stress-induced stretched-exponential time dependence of threshold voltage shift in InGaZnO thin film transistors. Appl. Phys. Lett. 93, 093504 (2008).

11. Lelis, A. J. et al. Time dependence of bias-stress-induced SiC MOSFET threshold-voltage instability measurements. IEEE Trans. Electron Devices 55, 1835-1840 (2008).

12. Lin, C., Chou, M., Kang, T. \& Wu, S. Electrical characteristics and TDDB breakdown mechanism of $\mathrm{N}_{2}$-RTA-treated Hf-based high-k gate dielectrics. Microelectron. Eng. 88, 950-958 (2011).

13. Linder, B. P., Lombardo, S., Stathis, J. H., Vayshenker, A. \& Frank, D. J. Voltage dependence of hard breakdown growth and the reliability implication in thin dielectrics. IEEE Electron Device Lett. 23, 661-663 (2002).

14. Wu, E. Y. \& Suñé, J. Power-law voltage acceleration: a key element for ultra-thin gate oxide reliability. Microelectron. Reliab. 45, 1809-1834 (2005).

15. Types of electrical overstress protection. ON Semiconductor AND9009/D https:// www.onsemi.com/pub/Collateral/AND9009-D.PDF (2014).

16. Wang, Z., Shi, X., Tolbert, L. M., Wang, F. \& Blalock, B. J. A di/dt feedback-based active gate driver for smart switching and fast overcurrent protection of IGBT modules. IEEE Trans. Power Electron. 29, 3720-3732 (2014).

17. Kwan, A. M. H. \& Chen, K. J. A gate overdrive protection technique for improved reliability in AIGaN/GaN enhancement-mode HEMTs. IEEE Electron Device Lett. 34, 30-32 (2013).

18. Chen, T. \& Ker, M. Investigation of the gate-driven effect and substrate-triggered effect on ESD robustness of CMOS devices. IEEE Trans. Dev. Mater. Reliab. 1, 190-203 (2001).

19. Sahin, H. et al. Anomalous Raman spectra and thickness-dependent electronic properties of WSe $\mathrm{W}_{2}$. Phys. Rev. B 87, 165409 (2013).

20. Mak, K. F., Lee, C., Hone, J., Shan, J. \& Heinz, T. F. Atomically thin $\mathrm{MoS}_{2}$ : a new direct-gap semiconductor. Phys. Rev. Lett. 105, 136805 (2010).

21. Chiu, M. et al. Determination of band alignment in the single-layer $\mathrm{MoS}_{2} / \mathrm{WSe}_{2}$ heterojunction. Nat. Commun. 6, 7666 (2015).

22. Chuang, $H$. et al. High mobility $\mathrm{WSe}_{2} \mathrm{p}$ - and n-type field-effect transistors contacted by highly doped graphene for low-resistance contacts. Nano Lett. 14, 3594-3601 (2014).

23. Kang, K. et al. High-mobility three-atom-thick semiconducting films with waferscale homogeneity. Nature 520, 656-660 (2015).

24. Bernardini, F., Fiorentini, V. \& Vanderbilt, D. Spontaneous polarization and piezoelectric constants of III-V nitrides. Phys. Rev. B 56, 10024-10027 (1997).

25. McDonnell, S. et al. $\mathrm{HfO}_{2}$ on $\mathrm{MoS}_{2}$ by atomic layer deposition: adsorption mechanisms and thickness scalability. ACS Nano 7, 10354-10361 (2013).

26. Lee, $C$. et al. Anomalous lattice vibrations of single- and few-layer MoS 2 . ACS Nano 4, 2695-2700 (2010)

27. English, C. D., Shine, G., Dorgan, V. E., Saraswat, K. C. \& Pop, E. Improved contacts to $\mathrm{MoS}_{2}$ transistors by ultra-high vacuum metal deposition. Nano Lett. 16, 3824-3830 (2016).

28. Chen, M. et al. TMD FinFET with $4 \mathrm{~nm}$ thin body and back gate control for future low power technology. IEDM Tech. Dig. 2015, 32.2.1-32.2.4 (2015).

29. Zhao, P. et al. 2D layered materials: from materials properties to device applications. IEDM Tech. Dig. 2015, 27.3.1-27.3.4 (2015).

30. Eichfeld, S. M. et al. Highly scalable, atomically thin $\mathrm{WSe}_{2}$ grown via metal-organic chemical vapor deposition. ACS Nano 9, 2080-2087 (2015).

31. Jeon, J. et al. Layer-controlled CVD growth of large-area two-dimensional $\mathrm{MoS}_{2}$ films. Nanoscale 7, 1688-1695 (2015).

32. Zhang, W. et al. High-gain phototransistors based on a CVD $\mathrm{MoS}_{2}$ monolayer. Adv. Mater. 25, 3456-3461 (2013).

33. $\mathrm{Yu}$, L. et al. High-performance $\mathrm{WS}_{2}$ complementary metal oxide semiconductor technology and integrated circuits. Nano Lett. 15, 4928-4934 (2015).

34. Das, S., Chen, H., Penumatcha, A. V. \& Appenzeller, J. High performance multilayer $\mathrm{MoS}_{2}$ transistors with scandium contacts. Nano Lett. 13, 100-105 (2013).

35. Sangwan, V. K. et al. Low-frequency electronic noise in single-layer $\operatorname{MoS}_{2}$ transistors. Nano Lett. 13, 4351-4355 (2013).

36. Park, W. et al. Oxygen environmental and passivation effects on molybdenum disulfide field effect transistors. Nanotechnology 24, 095202 (2013).

37. Qian, Q. et al. Enhanced dielectric deposition on single-layer $\mathrm{MoS}_{2}$ with low damage using remote $\mathrm{N}_{2}$ plasma treatment. Nanotechnology 28, 175202 (2017).

38. Azcatl, A. et al. $\mathrm{MoS}_{2}$ functionalization for ultra-thin atomic layer deposited dielectrics. Appl. Phys. Lett. 104, 111601 (2014).

39. Ma, N. \& Jena, D. Charge scattering and mobility in atomically thin semiconductors. Phys. Rev. X 4, 011043 (2014). 
40. $\mathrm{Yu}, \mathrm{Z}$. et al. Realization of room-temperature phonon-limited carrier transport in monolayer $\mathrm{MoS}_{2}$ by dielectric and carrier screening. Adv. Mater. 28, 547-552 (2016).

41. Seo, S., Jung, E., Chae, H. \& Cho, S. M. Optimization of $\mathrm{Al}_{2} \mathrm{O}_{3} / \mathrm{ZrO}_{2}$ nanolaminate structure for thin-film encapsulation of OLEDs. Org. Electron. 13, 2436-2441 (2012).

42. Allain, A., Kang, J., Banerjee, K. \& Kis, A. Electrical contacts to two-dimensional semiconductors. Nat. Mater. 14, 1195-1205 (2015).

43. $\mathrm{Li}, \mathrm{S}$. et al. Thickness scaling effect on interfacial barrier and electrical contact to two-dimensional $\mathrm{MoS}_{2}$ layers. ACS Nano 8, 12836-12842 (2014).

44. Li, L. et al. Black phosphorus field-effect transistors. Nat. Nanotechnol. 9, 372-377 (2014).

45. Wang, Y. et al. Field-effect transistors made from solution-grown two-dimensional tellurene. Nat. Electron. 1, 228-236 (2018).
Open Access This article is licensed under a Creative Commons Attribution 4.0 International License, which permits use, sharing, adaptation, distribution and reproduction in any medium or format, as long as you give appropriate credit to the original author(s) and the source, provide a link to the Creative Commons license, and indicate if changes were made. The images or other third party material in this article are included in the article's Creative Commons license, unless indicated otherwise in a credit line to the material. If material is not included in the article's Creative Commons license and your intended use is not permitted by statutory regulation or exceeds the permitted use, you will need to obtain permission directly from the copyright holder. To view a copy of this license, visit http://creativecommons. org/licenses/by/4.0/.

(c) The Author(s) 2019 